\title{
An Antibacterial Wound Dressing Based on GS-SF Composite Scaffold
}

\author{
Qifeng Jiang1, Ping Chen², Bin Gao ${ }^{1 *}$ \\ ${ }^{1}$ Department of Medical Information, Chongqing Medical University, Chongqing, China \\ ${ }^{2}$ Experimental Education Center, Chongqing Medical University, Chongqing, China \\ Email:^Anwell_J@163.com
}

How to cite this paper: Jiang, Q.F., Chen, P. and Gao, B. (2020) An Antibacterial Wound Dressing Based on GS-SF Composite Scaffold. Health, 12, 915-922. https://doi.org/10.4236/health.2020.128068

Received: July 16, 2020

Accepted: August 7, 2020

Published: August 10, 2020

Copyright $\odot 2020$ by author(s) and Scientific Research Publishing Inc. This work is licensed under the Creative Commons Attribution International License (CC BY 4.0).

http://creativecommons.org/licenses/by/4.0/

\section{Open Access}

\begin{abstract}
Although the treatment of burn wounds has made great progress, the incidence of wound infection is still the main cause of high mortality. In this study, a silk fibroin (SF) scaffold wound dressing incorporated with Gentamicin Sulfate (GS) was developed for the treatment of burn infected wounds, in which GS was used as anti-bacterial agent. GS was mixed with silk fibroin solution and then processed into GS-SF composite scaffold via electro-spinning. The results showed the scaffold exhibited uniform polyporous morphology with $80 \%$ porosity. Induced by methanol, the scaffold presented much improved mechanical properties and stability to protease $X I V$. More important, the scaffold presented significant growth inhibition on both Gram-positive (Staphylococcus aureus) and Gram-negative (Pseudomonas aeruginosa and Escherichia coli) bacteria.
\end{abstract}

\section{Keywords}

Silk Fibroin, Gentamicin Sulfate, Wound Dressing, Anti-Bacterial

\section{Introduction}

Skin is the largest organ of human body, which is composed of epidermis, dermis and subcutaneous adipose tissue. A complete skin surface is very important for the human body, which can maintain fluid balance and regulate the body temperature to protect the body from infection [1]. Burn infection is one of the most common and destructive forms of acute trauma. Because of bacterial infection, it prevents the wound from healing and may even lead to death, so throughout the history of human health, burn infection has always been a constant threat [2] [3]. Although artificial skin can replace the role of skin, solve the *Corresponding author. 
problem of insufficient healthy skin of patients and promote in the wound healing, the medical cost of artificial skin is often too higher for general patients to bear. So, it becomes more and more necessary to develop wound dressing scaffolds to reduce the incidence rate of burn infection and promote the wound healing of full-thickness defect.

Silk fibroin (SF) is a kind of hydrophobic natural polymer derived from silkworm cocoon [4]. Due to its rich source, low cost and many special properties, SF became a hot point in biomaterial area. Previous studies showed that SF exhibited tunable mechanical strength, thermal stability, as well as excellent biocompatibility and biodegradability [5]. In addition, due to the good processability of SF aqueous solution, it was often processed into microspheres, colloids, thin films and scaffolds [6] [7] [8]. For example, SF has been used as a biomaterial for cell culture in vitro and tissue engineering research in vivo [9]. Furthermore, SF contains peptide ingredients, which would promote the differentiation and proliferation of human skin fibroblasts. Thus, SF could be a unique candidate as wound dressing in skin wounds, especially for full-thickness burn wounds.

In this study, a silk fibroin scaffold wound dressing incorporated with Gentamicin sulfate (GS) was developed for the treatment of burn infected wounds, in which GS was used as anti-bacterial agent. GS was mixed with silk fibroin solution and then fabricated into GS-SF composite scaffold via electro-spinning. First, the inner structure of scaffold was probed with scanning electron microscopy (SEM); Second, the mechanical properties of scaffold were measured with universal testing machine; Third, protease $X I V$ assay was set up to test the stability of scaffold; At last, both Gram-positive (Staphylococcus aureus) and Gram-negative (Pseudomonas aeruginosa and Escherichia coli) bacteria were used to check out the anti-bacterial activities of the scaffold. The results showed that GS-SF wound dressing scaffold with sufficient porosity presented excellent mechanical properties and stability, with significant growth inhibition on both Gram-positive and Gram-negative bacteria, which indicated that GS-SF composite scaffold might provide a useful strategy for development of new wound dressing for burn wounds.

\section{Materials and Methods}

\subsection{Preparation of GS-SF Composite Scaffold}

The preparation of SF solution was mainly referred to previous studies [10]. Briefly, the small pieces of cocoons were boiled for $1 \mathrm{~h}$ in $\mathrm{Na}_{2} \mathrm{CO}_{3}$ solution of $0.02 \mathrm{~mol} / \mathrm{L}$ to remove sericin, and then washed with deionized water. After dry overnight at $60^{\circ} \mathrm{C}$, the SF was dissolved in $3 \mathrm{~mol} / \mathrm{L} \mathrm{LiBr}$ solution and heated in water bath at $60^{\circ} \mathrm{C}$ for $5 \mathrm{~h}$ and then dialyzed in a dialysis bag with a molecular weight of $10 \mathrm{kDa}$. The dialyzed SF solution was centrifuged at $5000 \mathrm{rpm} / 10 \mathrm{~min}$ then stored at $4^{\circ} \mathrm{C}$. $40 \mu \mathrm{l} \mathrm{GS}$ solution with the concentration of $50 \mathrm{mg} / \mathrm{ml}$ was mixed with $1 \mathrm{ml} \mathrm{SF}$ solution and then fabricated into GS-SF composite scaffold via electro-spinning. The scaffold was then induced with methanol for future 
application.

\subsection{Characterization of GS-SF Composite Scaffold}

The morphological characterization of GS-SF composite scaffold was performed with SEM with the accelerating voltage of $10 \mathrm{kV}$. Then, the scaffold was casted into uniform diameter and thickness $(12 \mathrm{~mm} \times 6 \mathrm{~mm})$, then the mechanical properties of scaffold were evaluated with universal testing machine to measure tensile strength.

\subsection{Measurement of Porosity of GS-SF Composite Scaffold}

The porosity measurement the scaffold was mainly referred to previous studies [11]. The beaker was filled with ethanol, and its mass W1 was weighed; the scaffold with mass $\mathrm{Wg}$, was immersed in ethanol for $2 \mathrm{~h}$ and the total weight was W2; After the sample full of ethanol was taken out, the remaining weight was W3. The calculation formula of porosity $\mathrm{P}$ is as follows: $P=(\mathrm{W} 2-\mathrm{W} 3-\mathrm{Wg}) /(\mathrm{W} 1-\mathrm{W} 3)$

\subsection{In Vitro Degradation of GS-SF Composite Scaffold}

In vitro degradation of scaffolds was mainly referred to previous studies [12]. At $37^{\circ} \mathrm{C}$ the scaffolds were immersed in $2 \mathrm{ml}$ of $0.5 \mathrm{mg} / \mathrm{ml}$ protease $X I V$ in phosphate buffer solution (PBS, $\mathrm{pH}=7.4$ ). Change the solution once a day. The control was immersed in PBS solution without protease XIV medium. Then, the scaffolds were rinsed with deionized water and placed at $37^{\circ} \mathrm{C}$ followed by the recording of mass change.

\subsection{Anti-Bacterial Activities of GS-SF Composite Scaffold}

The disc diffusion method was used to evaluate the anti-bacterial activities of GS-SF composite scaffold with both Gram-positive (Staphylococcus aureus) and Gram-negative (Pseudomonas aeruginosa and Escherichia coli) bacteria. Briefly, the bacterial was inoculated on the slope of the solid medium, and then incubated overnight at $37^{\circ} \mathrm{C}$, then rinsed with sterile water to form bacterial liquid. The bacteria liquid was mixed. The bacteria liquid was mixed and then applied evenly to the dish containing solid medium. The sterilized scaffolds which had been mixed with different concentration of GS were placed separately in the dish coated with bacteria. Finally, the measurement results were compared the half diameter difference between the ring and the scaffold [13].

\section{Results}

\subsection{The Uniform Porous Structure of GS-SF Composite Scaffold}

The morphological characterization of GS-SF composite scaffold was performed with SEM with the accelerating voltage of $5 \mathrm{kV}$. As shown in Figure 1, the scaffold presented uniform fibrous structures inside, the average diameter of fibers was around $5-10 \mu \mathrm{m}$. The fibers interwined with each other to form porous 
formation and the porosity rate was about $80 \%$.

\subsection{Methanol Treatment Promoted Mechanical Properties of GS-SF Composite Scaffold}

The previous studies have shown methanol treatment could increase content of $\beta$-sheet in SF structure, which is a key factor for stability of SF. After the treatment of methanol, the mechanical properties of scaffold were evaluated with universal testing machine to measure tensile strength. As shown in Table 1 and Figure 2, the Ultimate Tensile Strength (UTS) of scaffold induced by methanol increased from $75 \mathrm{KPa}$ to $140 \mathrm{KPa}$.

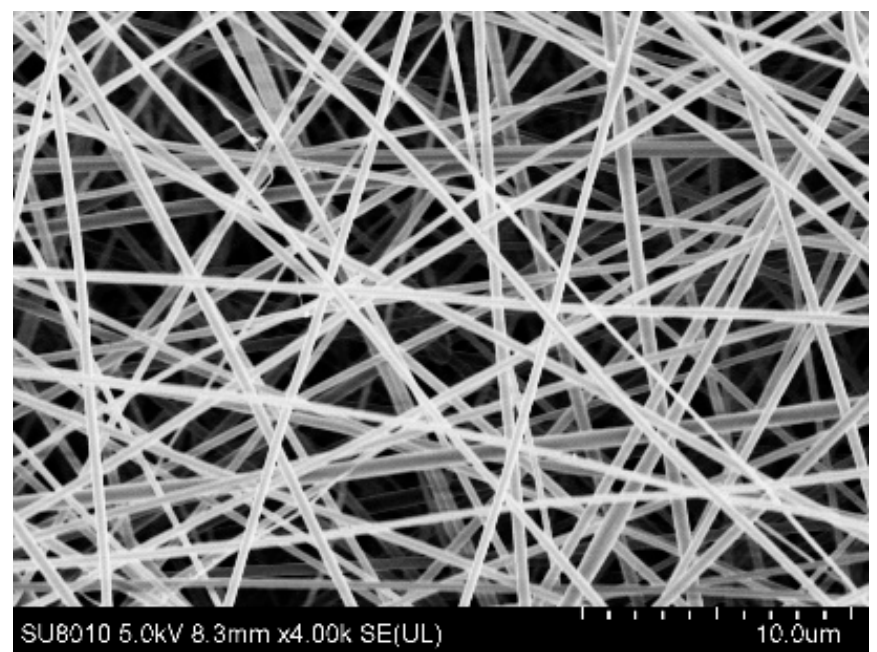

Figure 1. The SEM characterization of GS-SF composite scaffold: the scaffold was constructed with uniform fibers which formed porous structure, the average diameter of fibers was around $5-10 \mu \mathrm{m}$.

Table 1. Mechanical properties of GS-SF composite scaffold before and after methanol treatment.

\begin{tabular}{cc}
\hline & UTS \\
\hline Scaffold without methanol & $75 \pm 6.3 \mathrm{KPa}$ \\
Scaffold with methanol & $140 \pm 11.2 \mathrm{KPa}$ \\
\hline
\end{tabular}

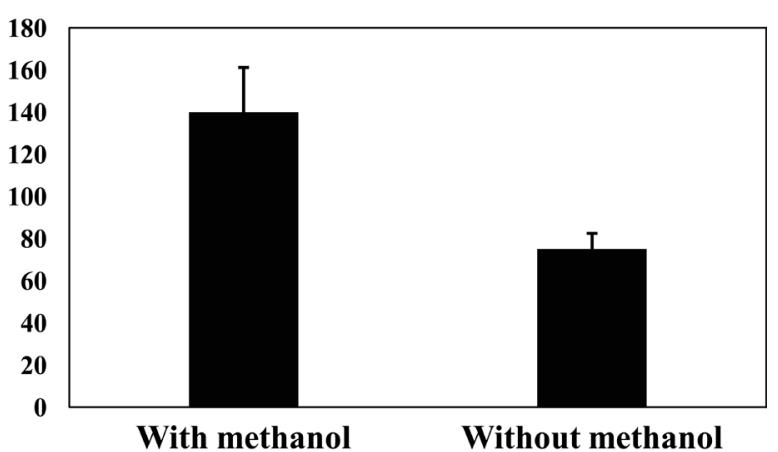

Figure 2. The UTS comparison of scaffolds before and after methanol treatment: methanol treatment significantly increased the UTS of scaffolds. 


\subsection{Methanol Treatment Decreased Degradation of GS-SF Composite Scaffold}

Degradation rate is an important factor to evaluate the quality of biological scaffolds which seriously affect the use value of the scaffold. Protease $X I V$ assay was set up to test the stability of scaffold. As shown in Figure 3, without methanol treatment, the scaffold degraded most rapidly in the first 3 days. By the fifth day, scaffold had been almost completely degraded. However, the degradation rate of scaffold induced by methanol was much slower, which remained $70 \%$ of the initial mass till ninth day.

\subsection{GS-SF Composite Scaffold Presented Significant Growth Inhibition on both Gram-Positive and Gram-Negative Bacteria}

The disc diffusion method was used to evaluate the anti-bacterial activities of GS-SF composite scaffold with both Gram-positive (Staphylococcus aureus) and Gram-negative (Pseudomonas aeruginosa and Escherichia coli) bacteria. The diameter of the antibacterial ring was used as the antibacterial activities of GS-SF scaffolds. As shown in Figure 4, there were significant antibacterial rings on the culture medium of the three kinds of bacteria in GS-SF composite scaffold. Besides, the diameter of the antibacterial rings increased with the increase of the concentration of GS. In the final concentration of $50 \mathrm{mg} / \mathrm{ml} \mathrm{GS}$, the diameter of the antibacterial ring was 5.5, 8.6 and $7.8 \mathrm{~mm}$ in $S$. aureus, $P$. aeruginosa and $E$. coli, respectively (Table 2 ).

\section{Discussion}

Burn, especially the extensive burn, is one of the most destructive forms of acute trauma to human body. Prevention of the infection in burn is the key step in clinical treatment. Traditional medical dressings based on other non-natural materials are not appropriate for all burn wounds types, due to immutability,

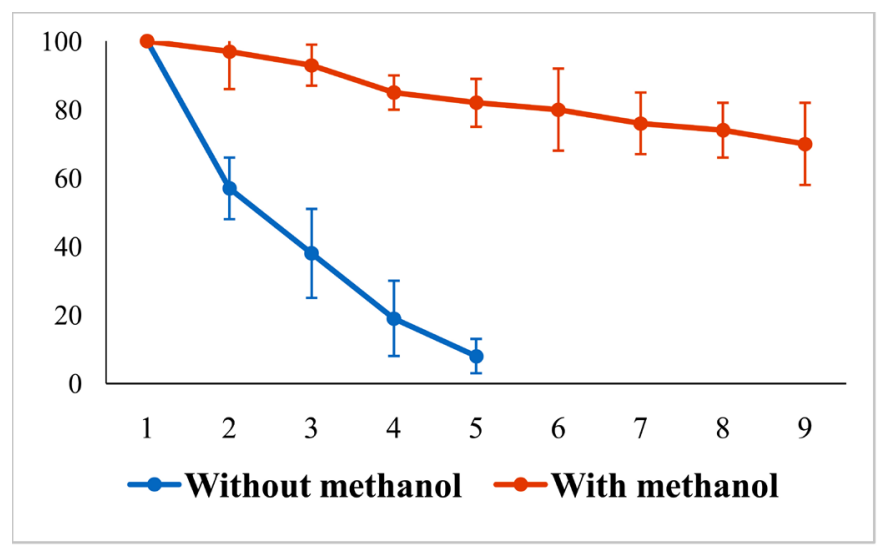

Figure 3. The comparison of degradation rate of the scaffolds before and after methanol treatment: the degradation rate was significantly decreased by methanol treatment; the scaffold remained $70 \%$ of the initial mass till the ninth day. The $\mathrm{x}$-axis represents time course of degradation in terms of day; the $y$-axis represents the percent of remaining mass of scaffolds. 

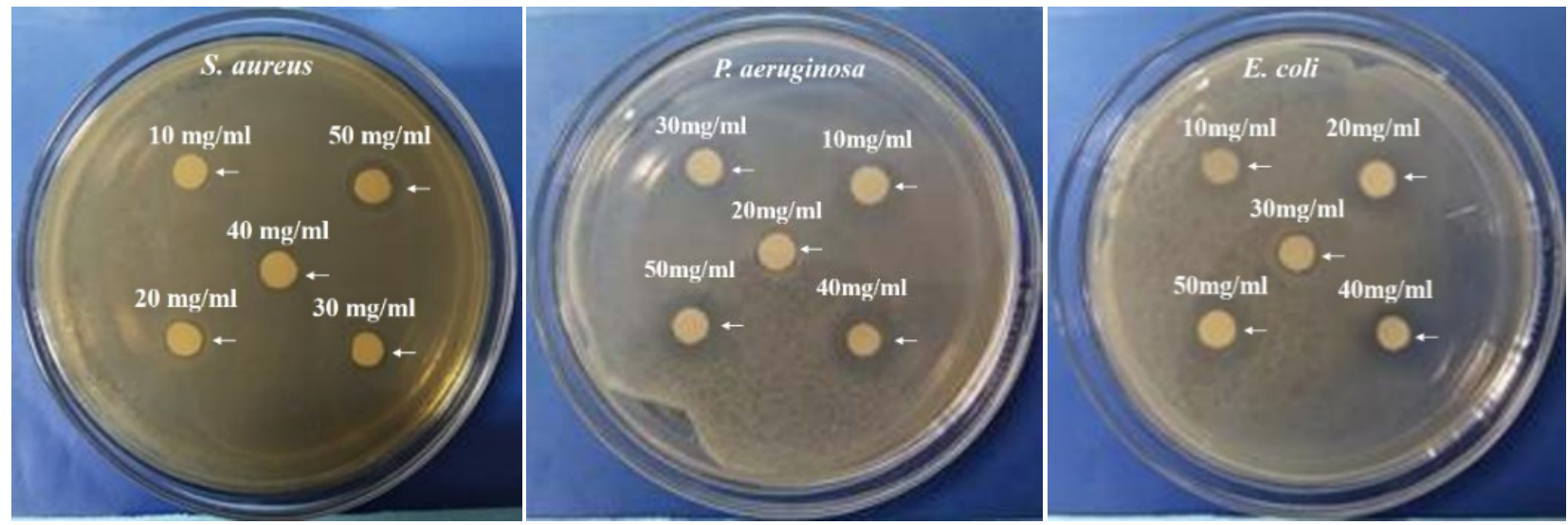

Figure 4. Anti-bacterial activities of scaffold against Gram-positive (Staphylococcus aureus) and Gram-negative (Pseudomonas aeruginosa and Escherichia coli) bacteria: the scaffolds showed significant inhibition on growth of bacteria which was indicated by antibacterial ring (white arrows). The diameter of antibacterial ring increased with the increase of GS concentration.

Table 2. The diameter of antibacterial ring in each bacterial with final concentration of 50 mg GS.

$\begin{array}{cc}\text { S. aureus } & 5.5 \mathrm{~mm} \\ \text { P. aeruginosa } & 8.6 \mathrm{~mm} \\ \text { E. coli } & 7.8 \mathrm{~mm}\end{array}$

non-bio-degradation and in-bio-compatibility. SF is a kind of natural protein mainly from cocoon and numerous studies have proved that SF proteins possess great biocompatibility to human [4] [5] [6], which gives SF high quality in wound dressing application. In this study, we presented a promising wound dressing for healing of the burn, which mainly consisted of SF protein and GS, in which the GS is a kind of common used antibiotics with little toxic effect with human body.

First, we considered the capacity of the drug loading in SF scaffold. As shown in Figure 1, with electro-spinning, the inner structure of scaffold was uniform nano-fibers with around $80 \%$ porosity rate, which indicated that sufficient GS could be loaded in this "wasp nest" structure; Second, the GS release rate was an important parameter for this wound dressing, which mainly affected by the degradation of the scaffold. With the methanol treatment, the degradation rate of scaffold significant decreased and this much slower degradation would change GS from burst release to form a kind of sustained release; Third, to meet different location of wound, tunable mechanical properties is another key factor for wound dressing. As shown in Figure 2, the pure SF scaffold was fragile with low UTS. With methanol treatment, we could significantly promote the UTS of SF scaffold to produce tougher scaffold; At last, GS is a kind of broad-spectrum antibiotics with extensive use. There were significant antibacterial rings on the culture medium of Staphylococcus aureus, Escherichia coli and Pseudomonas aeruginosa in GS-SF composite scaffold, which indicated that the GS-SF composite scaffolds presented significant growth inhibition on both Gram-positive and 
Gram-negative bacteria. All the results suggested that GS-SF composite scaffold might provide a useful strategy for development of new wound dressing for burn wounds.

\section{Conclusion}

In this study, we proposed a promising wound dressing based on GS-SF composite scaffolds. GS was efficient mixed with SF protein and fabricated into scaffold via electro-spinning. The inner structure of scaffold was constructed by crossing fibers with $80 \%$ porosity rate. The mechanical properties of scaffold could be tuned with methanol treatment as well as the degradation property, which indicated that this scaffold could be customized with different location of the wound. The scaffolds showed significant antibacterial activities to both Gram-positive and Gram-negative bacteria.

\section{Acknowledgements}

This research is supported by Chongqing Research Program of Basic Research and Frontier Technology (NO. Cstc2019jcyj-msxmX0164) and Science and Technology Research Program of Chongqing Municipal Education Commission (NO. KJQN201900414).

\section{Conflicts of Interest}

The authors declare no conflicts of interest regarding the publication of this paper.

\section{References}

[1] Church, D., Elsayed, S. and Reid, O. (2006) Burn Wound Infections. Clinical Microbiology Reviews, 19, 403-434. https://doi.org/10.1128/CMR.19.2.403-434.2006

[2] Salerian, A.J. (2020) Burn Wound Infections and Pseudomonas aeruginosa. Burns, 46, 257-258. https://doi.org/10.1016/j.burns.2019.07.008

[3] Rebecca, R., Adrien, P. and Melissa, B. (2019) Burn Wound Classification Model Using Spatial Frequency-Domain Imaging and Machine Learning. Journal of Biomedical Optics, 24, 1-9. https://doi.org/10.1117/1.JBO.24.5.056007

[4] Banani, K., Rangam, R., Subhas, C. and Wang, X.-G. (2013) Silk Fibroin Biomaterials for Tissue Regenerations. Advanced Drug Delivery Reviews, 65, 457-470. https://doi.org/10.1016/j.addr.2012.09.043

[5] Joydip, K., Laura, A., Poole, W., Penny, M. and Subhas, K. (2012) Silk Fibroin/Poly(Vinyl Alcohol) Photo-Crosslinked Hydrogels for Delivery of Macromolecular Drugs. Acta Biomaterialia, 8, 1720-1729. https://doi.org/10.1016/j.actbio.2012.01.004

[6] Vepari, C. and Kaplan, D.L. (2007) Silk as a Biomaterial. Progress in Polymer Science, 32, 991-1007. https://doi.org/10.1016/j.progpolymsci.2007.05.013

[7] Wenk, E., et al. (2008) Silk Fibroin Spheres as a Platform for Controlled Drug Delivery. Journal of Controlled Release, 132, 26-34. https://doi.org/10.1016/j.jconrel.2008.08.005

[8] Nicholas, G., Annie, B., Bernardo, P. and Kaplan, D.L. (2011) Lyophilized Silk Fi- 
broin Hydrogels for the Sustained Local Delivery of Therapeutic Monoclonal Antibodies. Biomaterials, 32, 2642-2650.

https://doi.org/10.1016/j.biomaterials.2010.12.023

[9] Wang, Y.-Z., Kim, H.-J., Gordana, N. and Kaplan, D.L. (2006) Stem Cell-Based Tissue Engineering with Silk Biomaterials. Biomaterials, 27, 6064-6082.

https://doi.org/10.1016/j.biomaterials.2006.07.008

[10] Joydip, K., Moumita, D., Sarani, G. and Kundu, S.C. (2008) Mulberry Non-Engineered Silk Gland Protein Vis-a-Vis Silk Cocoon Protein Engineered by Silkworms as Biomaterial Matrices. Journal of Materials Science: Materials in Medicine, 19, 2679-2689. https://doi.org/10.1007/s10856-008-3398-1

[11] She, H.-D., Xiao, X.-F. and Liu, R.-F. (2007) Preparation and Characterization of Polycaprolactone-Chitosan Composites for Tissue Engineering Applications. Journal of Material Science, 42, 8113-8119. https://doi.org/10.1007/s10853-007-1706-7

[12] Panya, W., Hiroki, U., Yasuhiko, T. and Ratana, R. (2010) In Vitro and in Vivo Release of Basic Fibroblast Growth Factor Using a Silk Fibroin Scaffold as Delivery Carrier. Journal of Biomaterials Science, Polymer Edition, 21, 1403-1419. https://doi.org/10.1163/092050609X12517858243706

[13] Wei, B., Yang, G. and Hong, F. (2011) Preparation and Evaluation of a Kind of Bacterial Cellulose Dry Films with Antibacterial Properties. Carbohydrate Polymers, 84, 533-538. https://doi.org/10.1016/j.carbpol.2010.12.017 